\title{
Make Urine Great Again
}

\author{
Nuno Moreira Fonseca ${ }^{1}$, David Navarro ${ }^{1}$, Giuseppe Garigali², Giovanni Battista Fogazzi ${ }^{2}$ \\ ${ }^{1}$ Nephrology Department, Centro Hospitalar Universitário de Lisboa Central, Hospital Curry Cabral, Lisbon, Portugal. \\ ${ }^{2}$ Clinical and Research Laboratory on Urinary Sediment, U.O.C. di Nefrologia, Dialisi e Trapianto di Rene, Fondazione IRCCS Ca' Granda Ospedale Maggiore Policlinico, Milano, Italy.
}

Microscopic examination of urinary sediment (U-sed) is an affordable and reliable diagnostic tool that constitutes a cornerstone of renal care. It provides essential information for the understanding of the pathophysiology of kidney injury. In recent decades it has become increasingly rare for this exam be performed by nephrologists, mainly due to the widespread diffusion of automated technologies and centralization of laboratory testing. The growing reliance on automated technology set the current path of continuous loss of nephrologists' skills and training opportunities for U-sed microscopy.

Automated U-sed evaluation is an attractive option since it increases considerably the number of samples that can be analysed at the same time, requiring less trained personnel. This is a crucial advantage for any institution processing a large number of samples. Nevertheless, manual examination remains the gold standard for automated analysis. ${ }^{1}$ Manual analysis offers substantial information that goes unrecognized with automated evaluation, particularly in the setting of acute kidney injury, hematuria and nephrolithiasis. Nephrology patients stand to benefit the most from manual examination, especially when it is performed by providers who can integrate clinical history, physical examination, kidney imaging and other laboratory testing to better assist the diagnosis and management of kidney disease.

The usefulness of U-sed microscopy is paradigmatic in the setting of acute kidney injury (AKI) evaluation due to its heterogeneous pathophysiology, elevated frequency and associated morbidity and mortality. $\mathrm{U}$-sed analysis is helpful in differentiating prerenal AKI from AKI with acute tubular necrosis (ATN). This distinction is important as prognosis and management differ, thus making early clinical differentiation vital. U-sed findings in ATN are conspicuous and include the presence of renal tubular epithelial cells (RTEC), RTEC casts, and granular casts. ${ }^{2}$ Pre-renal AKI presents findings which could be considered more bland, such as hyaline and granular casts. ${ }^{2} \mathrm{U}$-sed microscopy as part of the clinical workup of kidney disease is also useful for the assessment of other etiologies of AKI, particularly acute glomerulonephritis (RTEC, RTEC casts, and granular casts plus a significant number of dysmorphic erythrocytes and RBC casts), acute interstitial nephritis (RTEC, RTEC casts, and granular casts plus a leukocytes and leukocyte casts). Although the practice of checking urinary eosinophils in patients suspected of having AIN has become widespread, it is a test that performs poorly and may lead to a false positive diagnosis with inappropriate use of steroids to treat AIN or inappropriate changes in therapy. ${ }^{3}$ Lipidura can also be confirmed with the finding of lipid droplets, which are seen as a Maltese cross under polarized light. (Figure 1)

Isolated microscopic hematuria is also a very frequent motive of patient referral to nephrologists. Performing erythrocyte morphology allows for the distinction of origin of hematuria: glomerular vs. non-glomerular. Red blood cells can be classified as isomorphic (Figure 2), dysmorphic (Figure 3), these last including also acanthocytes (Figure

\section{Figure 1}

Lipid droplets (on the left,400x phase-contrast), showing a Maltese cross shape (on the right, 400x polarized light)

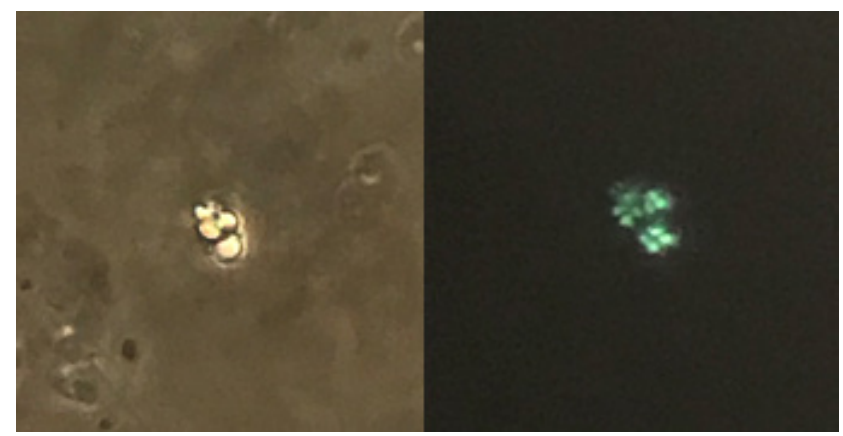

\section{Figure 2}

Isomorphic red blood cells (400x phase-contrast)

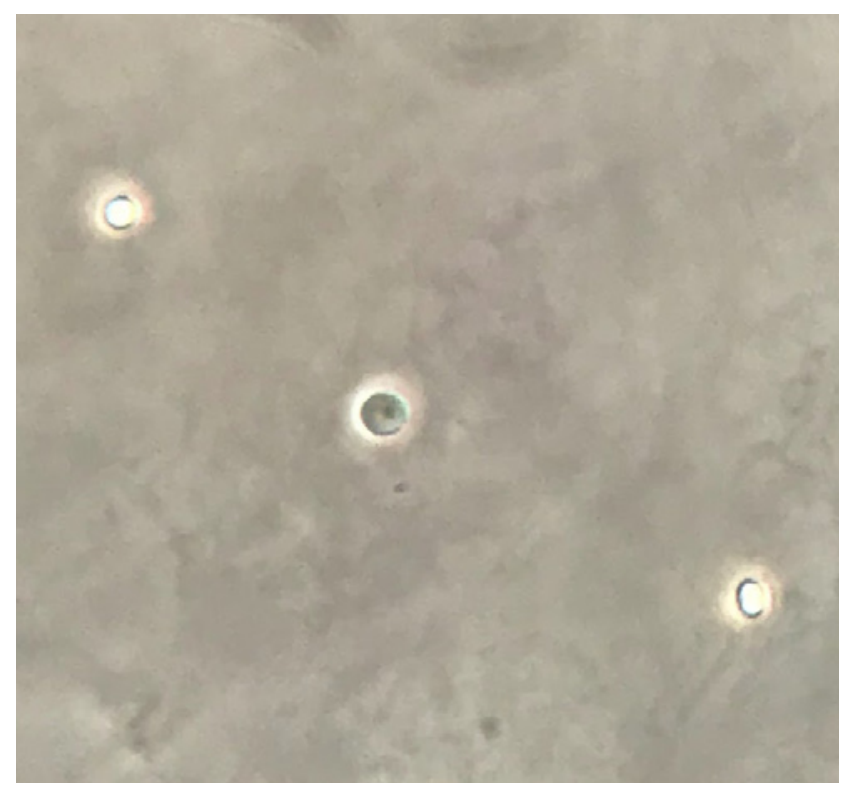




\section{Figure 3}

Dysmorphic red blood cells (400x phase-contrast)

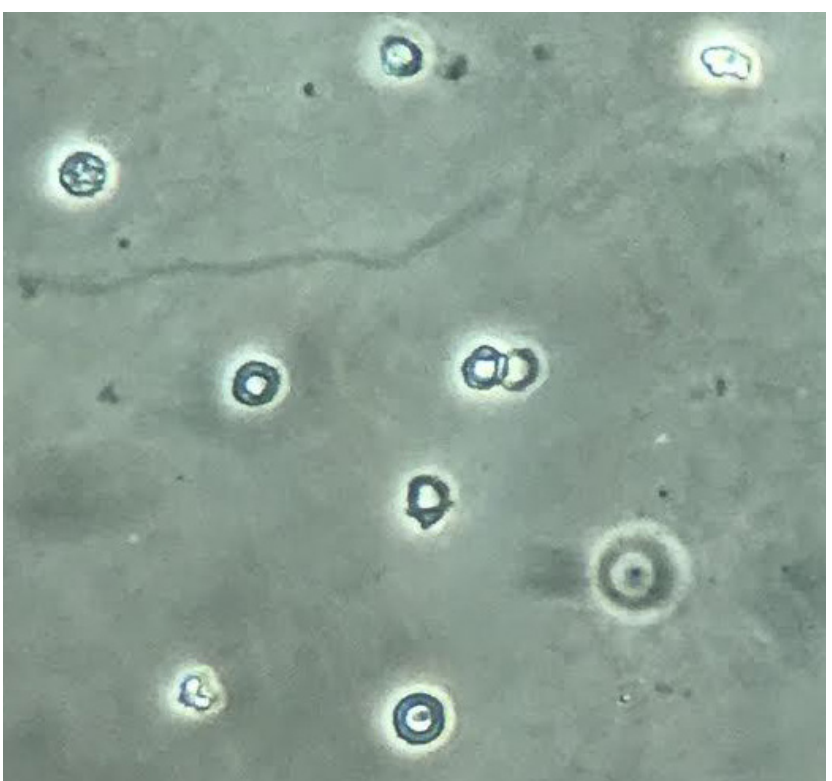

\section{Figure 4}

Acanthocyte (center right), Isomorphic red blood cells (center left) (400x phase-contrast)

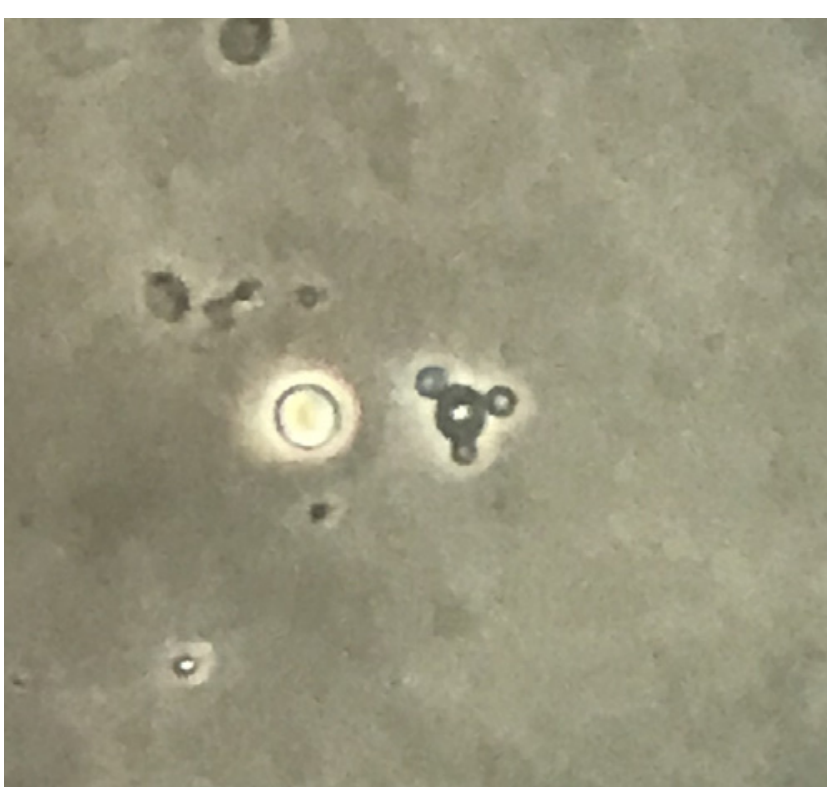

4). Acanthocytes, also known as G1 cells, are doughnut-shaped dysmorphic erythrocytes with one or more vesicle-like protrusions which can be identified easily and with much less subjectivity than other dysmorphic erythrocytes. For these reasons the threshold for defining

\section{Figure 5}

Red blood cell cast (400x phase-contrast)

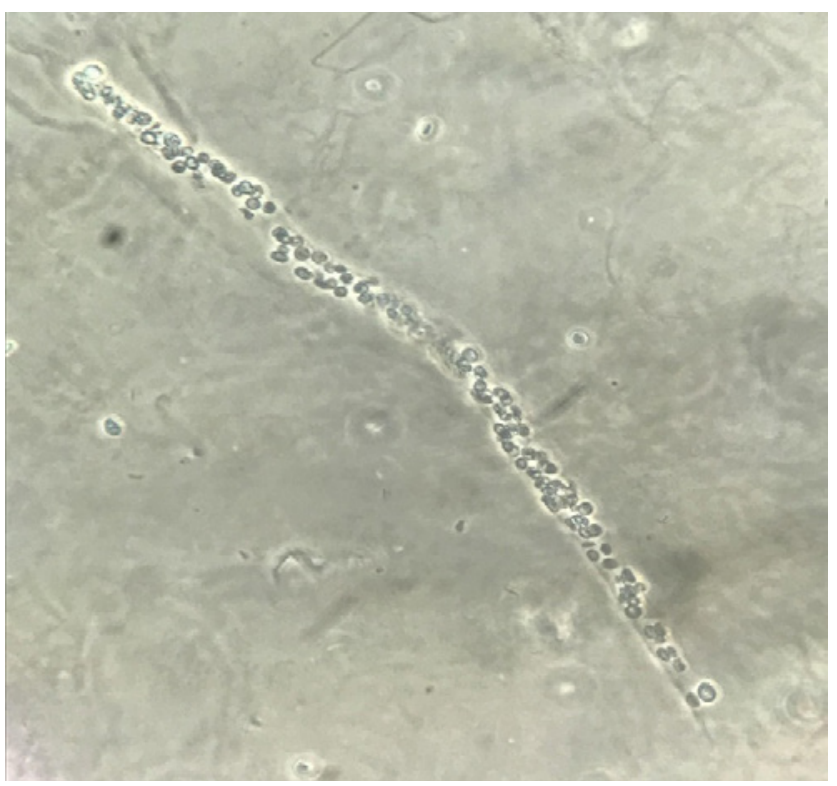

\section{Figure 6}

Uric acid crystals (400x polarized light)

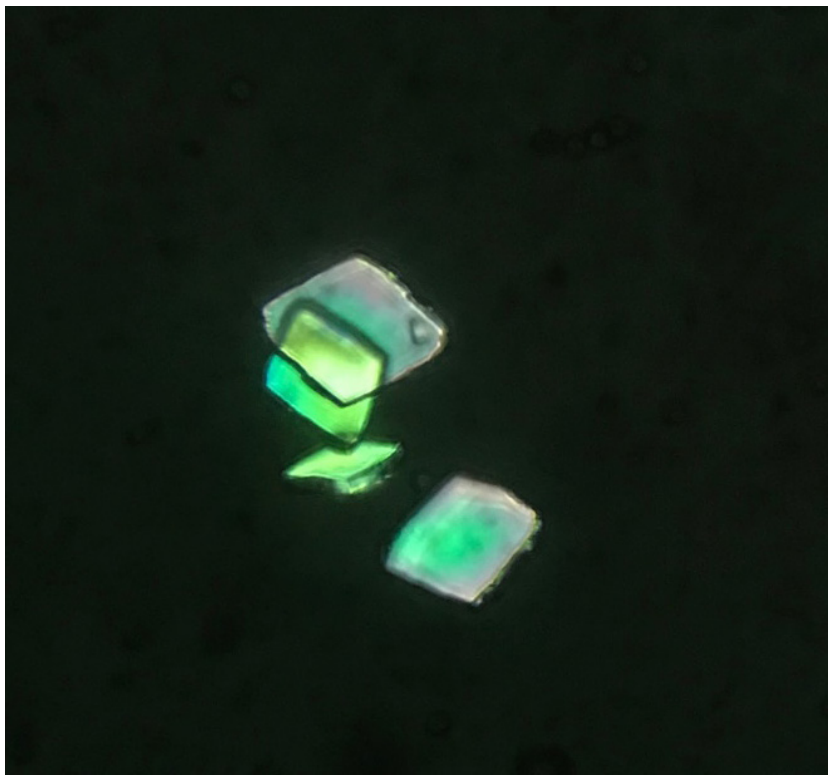

glomerular hematuria is lower when compared to non-acanthocyte-dysmorphic RBCs (>5\% vs. $>40 \%$ ). Dysmorphic RBCs and RBC casts (Figure 5) are signs of glomerular damage and are a frequent finding in glomerulonephritis. 


\section{Figure 7}

Decoy cell (400x phase-contrast)

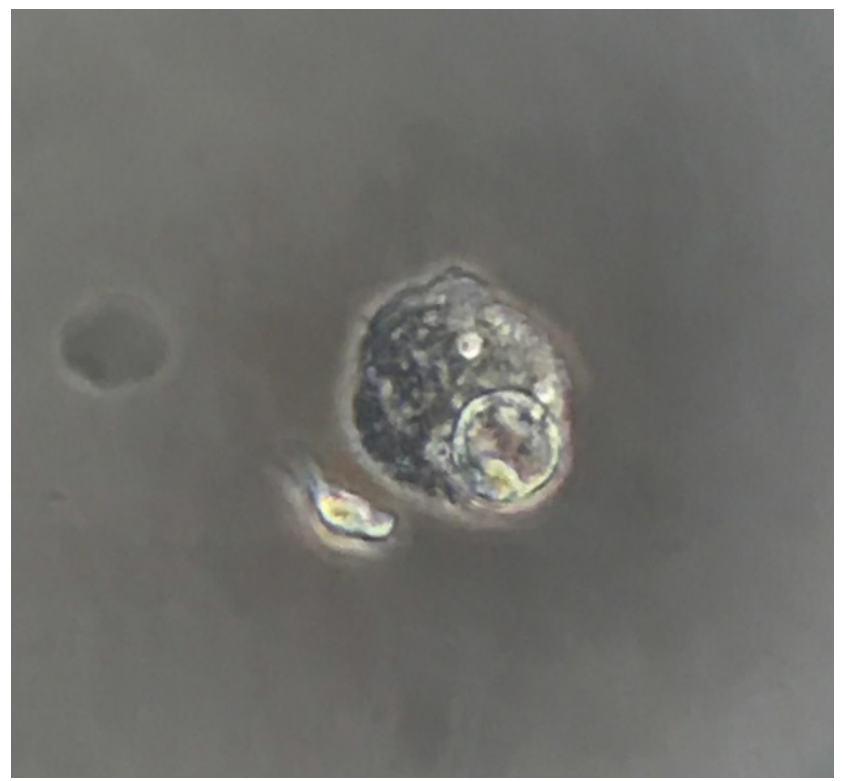

U-sed examination can be a helpful tool for nephrologists in the follow-up of an ongoing glomerular injury or in the identification of its recurrence. ${ }^{4}$

Isomorphic RBCs can be present in the setting of renal extraglomerular conditions such as acute interstitial nephritis, or urological diseases such as kidney and urinary tract neoplasia, urinary tract infection, or nephrolithiasis. Overall, RBC morphology may be helpful to guide clinical decision regarding further hematuria evaluation: kidney biopsy or serum immunological testing for glomerular hematuria versus urology referral for non-glomerular hematuria.

Crystalluria is a surrogate of urine supersaturation with substances derived from metabolic disorders, inherited diseases or drugs. Crystal identification requires knowledge of crystal morphologies, urine $\mathrm{pH}$, and birefringence features under polarized light microscopy. The main urinary crystalline categories include calcium oxalate, uric acid (Figure 6), struvite, calcium phosphate, cystine, and drugs (e.g. sulfamethoxazole, amoxicillin, ciprofloxacin, atazanavir). ${ }^{5}$ In the setting of AKI, U-sed examination can be instrumental for clinical decision-making by supporting withdrawal of an offending drug when its crystalline form is found in U-sed examination. Crystalluria also plays multiple key roles in the evaluation and management of nephrolithiasis as it can help determine the etiology of nephrolithiasis, especially in the absence of kidney stone analysis. It is also useful to determine the activity of stone disease and its response to therapeutic measures in the follow-up of patients. ${ }^{6}$

The utility of $\mathrm{U}$-sed also includes transplant nephrology. Polyomavirus nephropathy (PVN) is a common opportunistic viral infection of renal allografts with most cases occurring in the first year after transplantation. Decoy cells found in urine result from polyomavirus infection and present with an irregular enlarged nuclei, altered chromatin, cytoplasmic vacuoles and the presence of a halo (Figure 7). ${ }^{7}$ Routine search for urinary decoy cells is an effective screening method for PVN since this test has a negative predictive value of $100 \%$, ruling out all negative cases. ${ }^{8} \mathrm{~A}$ positive result will require an evaluation of PV viremia, as the positive predictive value (PPV) of viremia is superior to that of decoy cell search (50-60\% vs $29 \%$ ). Notwithstanding, a definitive diagnosis of PVN requires histological confirmation via renal biopsy. ${ }^{9}$

If U-sed analysis can bring such a wealth of information to the avid mind of the clinical nephrologist, what does it take to include it in daily practice? Proper equipment and training. A major barrier can be the access to a phase-contrast microscope.

Most hospitals already have bright field microscopy with polarized light as well as centrifuges, meaning that $U$-sed microscopy can already be performed without any added cost. Ideally phase-contrast microscopes should be used, and although these have become increasingly rare in most institutions, it is worth inquiring if another department in the hospital has one. ${ }^{10}$ If available microscopes are not equipped with integrated cameras to capture the images of U-sed microscopy, the advent of smartphones has made it possible to take high-quality pictures. ${ }^{11}$

Acquiring competency in U-sed examination requires training. Ideally this should be provided by experienced nephrologists in a laboratory with large sample volume. Most departments can implement these by having a referral fellow or nephrologist training extra muros. In our department we have successfully implemented a U-sed laboratory after two fellows (NMF, DN) trained for 4 weeks at major laboratory with experts (GG, GBF). Training encompasses use of a standardized method for urine collection and handling, correct identification of the most important particles of U-sed, and knowledge of their clinical meaning. ${ }^{12}$

In addition to clinical utility, U-sed examination attractiveness also resides in the avenues it opens for clinical nephrology research. U-Sed examination is a critical diagnostic tool for diagnosis and bedside care of nephrology patients. It is an inexpensive and relatively simple diagnostic tool, which supplies crucial information. Physician-performed urine microscopy is empowered by integration of microscopy findings with clinical data. It is time to reclaim nephrology's oldest knowledge and make urine great again.

\section{Disclosure of potential conflicts of interest: none declared}

\section{References}

1. Cavanaugh C, Perazella MA. Urine sediment examination in the diagnosis and management of kidney disease: core curriculum 2019. Am J Kidney Dis. 2019;73(2):258-72.

2. Perazella MA, Coca SG, Kanbay M, Brewster UC, Parikh CR. Diagnostic value of urine microscopy for differential diagnosis of acute kidney injury in hospitalized patients. Clin J Am Soc Nephrol. 2008;3(6):1615-9.

3. Muriithi AK, Nasr SH, Leung N. Utility of urine eosinophils in the diagnosis of acute interstitial nephritis. Clin J Am Soc Nephrol. 2013;8(11):1857-62.

4. Rhee RL, Davis JC, Ding L, Fervenza FC, Hoffman GS, Kallenberg CG, Langford CA, McCune WJ, Monach PA, Seo P, Spiera R. The utility of urinalysis in determining the risk of renal relapse in ANCA-associated vasculitis. Clin J Am Soc Nephrol. 2018;13(2):251-7. 
5. Verdesca S, Fogazzi GB, Garigali G, Messa P, Daudon M. Crystalluria: prevalence, different types of crystals and the role of infrared spectroscopy. Clin Chem Lab Med. 2011;49(3):515-20.

6. Daudon M, Frochot V, Bazin D, Jungers P. Crystalluria analysis improves significantly etiologic diagnosis and therapeutic monitoring of nephrolithiasis. Comptes Rendus Chimie. 2016;19(11-12):1514-26.

7. Hirsch HH, Knowles W, Dickenmann M, Passweg J, Klimkait T, Mihatsch MJ, Steiger J. Prospective study of polyomavirus type BK replication and nephropathy in renal-transplant recipients. N Engl J Med. 2002;347(7):488-96.

8. Fogazzi GB, Cantù M, Saglimbeni L. 'Decoy cells' in the urine due to polyomavirus BK infection: easily seen by phase-contrast microscopy. Nephrol Dial Transplant 2001;16:1496-8.

9. Fonseca NM, Navarro D, Góis M, Sousa H, Aires I, Nolasco F. Streamlining diagnosis with urinary sediment microscopy: a case of deteriorating renal function in a kidney transplant patient. Port $J$ Nephrol Hypert. 2018;32(4):389-92.

10. Fogazzi GB, Delanghe J. Microscopic examination of urine sediment: Phase contrast versus bright field. Clin Chim Acta. 2018;487:168-73.
11. Fogazzi GB, Garigali G. The different ways to obtain digital images of urine microscopy findings: Their advantages and limitations. Clin Chim Acta. 2017;466:160-1.

12. Fogazzi GB, Verdesca S, Garigali G. Urinalysis: core curriculum 2008. Am J Kidney Dis. 2008;51(6):1052-67.

\section{Correspondence to:}

Nuno Moreira Fonseca, MD, MA.

Department of Nephrology, Centro Hospitalar Universitário de Lisboa Central, Rua da Beneficência 8, 1069-166 Lisbon, Portugal.

E-mail: nuno.mf@nyu.edu 